Agron. Mesoam. 29(1):85-93. Enero-abril, 2018

ISSN 2215-3608, doi:10.15517/ma.v29i1.28053

http://www.revistas.ucr.ac.cr/index.php/agromeso

\title{
Abundancia y cobertura de arvenses bajo manejo convencional y orgánico de café y banano ${ }^{1}$
}

\section{Weed abundance and ground cover under conventional or organic management of coffee and banana}

\author{
Renán Agüero-Alvarado², Ana María Rodríguez-Ruiz², María Isabel González-Lutż, Pamela Portuguez-García², \\ Steven Brenes-Prendas ${ }^{4}$
}

\begin{abstract}
Resumen
Ciertas especies de la vegetación espontánea pueden ser utilizadas como cobertura en cultivos como banano y café. El objetivo de este estudio fue comparar la cobertura y la abundancia de arvenses en los sistemas productivos orgánico y convencional de banano y café. Los muestreos de la vegetación se realizaron entre los años 2015 y 2016 en fincas seleccionadas de las provincias de Limón y Alajuela, Costa Rica. Se usó la metodología de área mínima para determinar el tamaño de los sitios muestrales. En cada sitio se identificaron todas las especies de arvenses presentes y se agruparon por familia. También se determinó la cobertura porcentual de cada especie, por medio de una escala visual. En ambos cultivos, la diversidad de arvenses fue mayor cuando se usó control químico con glifosato, mientras que la cobertura máxima de cada especie tendió a mantenerse al mínimo de la escala de evaluación, cuando se asperjó ese herbicida. Bajo el manejo orgánico con chapias, se estableció una jerarquía competitiva de especies dominantes, que no permitió el establecimiento de otras menos adaptadas al sitio; en contraste, el uso de herbicidas, si redujo significativamente las poblaciones de arvenses, dejó abiertos abundantes espacios para que un mayor número de especies se regeneren a partir del banco de propágulos. El manejo orgánico en ambos cultivos mostró mayor cobertura de arvenses en el suelo, pero en menor cantidad de especies. En contraste, el manejo químico con glifosato mostró un mayor número de especies, pero una significativa reducción en la cobertura del suelo por arvenses.
\end{abstract}

Palabras clave: cultivos perennes, coberturas naturales, manejo de arvenses, glifosato, cobertura máxima.

\begin{abstract}
Certain weeds can be used as cover species in bananas and coffee plantations. The objective of this study was to compare ground cover and abundance of weed species, between the organic and conventional production systems of banana and coffee. Selected farms under organic or conventional management located at Limón and Alajuela were surveyed for weeds, during 2015 and 2016. The minimum area methodology was used to determine sampling site size. At each sampling site, all weed species were identified and grouped into their respective taxonomic families.
\end{abstract}

\footnotetext{
Recibido: 8 de marzo, 2017. Aceptado: 4 de mayo, 2017. Este trabajo formó parte del proyecto 736-B3-164 inscrito en la Vicerrectoría de Investigación de la Universidad de Costa Rica, Estación Experimental Fabio Baudrit Moreno, y del proyecto ED-1816 inscrito en la Vicerrectoría de Acción social, Universidad de Costa Rica. San José, Costa Rica.

2 Universidad de Costa Rica, Estación Experimental Fabio Baudrit Moreno. Alajuela, Costa Rica. ragueroster@gmail.com, amrodriguezster@ gmail.com,mapapo69@gmail.com

3 Universidad de Costa Rica, Escuela de Estadística. San José, Costa Rica. mariaisabel.gonzalezlutz@ucr.ac.cr

4 Universidad Escuela de Agricultura de la Región Tropical Húmeda (EARTH). Limón, Costa Rica. sbrenes@earth.ac.cr
} 
Percent soil cover by each species was also estimated by means of a visual scale. In both crops, weed diversity was higher when the herbicide glyphosate was used for weed control, although the ground cover for these species was kept at a minimum. These results are thought to arise from the fact that under the organic production system, a hierarchy of dominant species is established, which in turn inhibit many of the somewhat less adapted species, whereas under the conventional production system, the use of a non-residual herbicide renders the ground with plenty of space for species regeneration from the soil propagule bank. Organic weed management in both crops showed higher soil cover by weeds, but from a lower number of species. In contrast, chemical weed management with glyphosate showed a higher number at species, but a significant reduction in ground cover by those weeds.

Keywords: perennial crops, natural covers, weed management, glyphosate, maximum ground cover.

\section{Introducción}

El agroecosistema bananero permite el desarrollo de una gran diversidad de flora asociada al cultivo (Brenes, 2005; Agüero y Brenes, 2015). Dentro de esta flora existen especies con un alto potencial para ser utilizadas como coberturas espontáneas, por sus características morfológicas y agronómicas. Entre las principales características de las arvenses de cobertura para el cultivo de banano están: que tengan un porte bajo no mayor a $10 \mathrm{~cm}$ de altura, que cubran bien el suelo, que soporten el pisoteo y la sombra, que su sistema radical no tenga una profundidad mayor a $5 \mathrm{~cm}$, que su hábito de crecimiento no sea trepador y que no sean hospederos alternos de plagas que afecten al cultivo (Wielemaker, 1997; Cubillo et al., 2001; Pinilla y García, 2002a). Con el uso de estas coberturas se busca mejorar las condiciones de producción del cultivo, por los múltiples beneficios que estas arvenses pueden ofrecer al sistema.

Entre algunos de los beneficios que las coberturas aportan al sistema productivo de banano se encuentran: la disminución de la erosión y pérdida de suelo, disminución en la temperatura superficial del suelo, aumento en la microfauna y salud del suelo, disminución de la lixiviación de los nutrientes aportados por los fertilizantes, aporte de materia orgánica, disminución de sedimentos en los canales de drenaje (De-la-Cruz et al., 2001; Pinilla y García, 2002b; Carbonó y Cruz, 2005). Además, se considera que al utilizar una policobertura, es decir, permitir que varias de estas arvenses con potencial de cobertura se desarrollen juntas, se obtiene un agroecosistema más equilibrado y sostenible, ya que será más difícil que una especie agresiva y competitiva domine y cause problemas en el crecimiento y desarrollo del cultivo (Garro, 2002).

Para el cultivo de café, se reporta vegetación espontánea que podría usarse como cobertura noble (Herrera, 1997), porque posee un sistema radical superficial, un crecimiento reducido en altura, y porque su capacidad de cubrimiento no interfiere en el rendimiento del café (Hincapié-Gómez y Salazar-Gutiérrez, 2007). El uso de coberturas en café constituye una herramienta de conservación del suelo y de agua para mejorar la sostenibilidad del cultivo. Otro beneficio es que, permiten un manejo integrado de arvenses, dado que las coberturas pueden desplazar el crecimiento de arvenses de difícil manejo (Salazar et al., 2012).

El objetivo de este trabajo fue comparar la cobertura y la abundancia de arvenses en los sistemas productivos orgánico y convencional de banano y café.

\section{Materiales y métodos}

Entre los años 2015 y 2016, se evaluó la flora asociada a los cultivos de banano (en dos fincas) y de café (en cuatro fincas), que poseían dos sistemas de producción (orgánico y convencional). Para ambos cultivos, bajo la 
producción convencional el manejo de arvenses se basó en el control químico mediante la aplicación de glifosato a razón de $960 \mathrm{~g}$ ia/ha, aplicado en café dos veces al año a un intervalo de aproximadamente seis meses cada aplicación, y hasta seis ciclos de aspersión en banano; mientras que en la producción orgánica de ambos cultivos se emplearon chapeas. El levantamiento de arvenses en banano bajo el sistema de producción orgánica se hizo en la finca de la EARTH (Escuela de Agricultura de la Región Tropical Húmeda), ubicada entre las coordenadas $10^{\circ} 197049$ y $83^{\circ} 596792$ en el cantón de Guácimo, en la provincia de Limón, Costa Rica. El cantón posee una altitud de $114 \mathrm{msnm}$, una temperatura promedio de $26,2^{\circ} \mathrm{C}$ (mínima) y $31,6^{\circ} \mathrm{C}$ (máxima) y una precipitación de 4023,2 mm (IMN, 2002). El levantamiento bajo el sistema de producción convencional en banano se efectuó en una finca denominada Agrotubérculos ubicada en el cantón de Talamanca, en la provincia de Limón. El cantón se encuentra a $29 \mathrm{msnm}$, con una temperatura promedio de $24,5^{\circ} \mathrm{C}$ (mínima) y $28,6{ }^{\circ} \mathrm{C}$ (máxima) y una precipitación de 3082,1 mm (IMN, 2002). Para hacer el levantamiento se siguió la metodología del área mínima citada por Jürgens (1985). En cada finca se recorrieron de uno a tres domos y se escogieron al azar diez sitios muestreales con un tamaño de $100 \mathrm{~m}^{2}$ cada sitio (Cuadro 1).

Cuadro 1. Sitios de muestreo de arvenses en café y banano, bajo dos sistemas de producción. Limón y Alajuela, Costa Rica. 20152016.

Table 1. Weed surveying sites in coffee and banana crops under two production systems. Limón and Alajuela, Costa Rica. 20152016.

\begin{tabular}{cccc}
\hline Cultivo & Sistema productivo & Número de Fincas & Número de sitios \\
\hline \multirow{2}{*}{ Café } & Convencional & 2 & 28 \\
& Orgánico & 2 & 28 \\
\hline \multirow{2}{*}{ Banano } & Convencional & 1 & 10 \\
& Orgánico & 1 & 10 \\
\hline
\end{tabular}

Las fincas de producción de café bajo manejo orgánico y convencional escogidas, se ubicaron en el cantón de Naranjo en la provincia de Alajuela, el cual se sitúa a $1054 \mathrm{msnm}$, con una temperatura promedio de 21,4 ${ }^{\circ} \mathrm{C}$ (mínima) y $26,2{ }^{\circ} \mathrm{C}$ (máxima) y con una precipitación de $2409 \mathrm{~mm}$ (IMN, 2002). Las fincas con manejo convencional se ubicaron entre las coordenadas $10^{\circ} 16602-84^{\circ} 38963$ y $10^{\circ} 16665-84^{\circ} 38706$ y las de manejo orgánico entre $10^{\circ} 15890-84^{\circ} 38825$ y $10^{\circ} 168765-84^{\circ} 38337$. Para llevar a cabo el levantamiento se escogieron al azar siete lotes, en cada uno de los cuales se estableció un sitio muestral de $100 \mathrm{~m}^{2}$, por medio de la metodología mencionada anteriormente (Cuadro 1).

Las especies que no fueron identificadas in situ, se colectaron y fueron identificadas en el Laboratorio de Arvenses de la Universidad de Costa Rica y en la EARTH. Posteriormente, se realizó el conteo de las mismas y se agruparon por familia en el programa Excel 2007, para obtener la variable abundancia de las familias taxonómicas.

El diseño experimental fue un arreglo factorial de dos factores (tipo de cultivo y sistema productivo) a dos niveles cada uno (Cuadro 1).

Para la medición de la cobertura de las arvenses, se hizo una evaluación visual con la escala que se muestra en el Cuadro 2.

Se reportan los datos de cobertura máxima, por reflejar estos las especies con mayor potencial de dominar el sitio. Para hacer el cálculo de los promedios de cobertura se utilizaron los puntos medios de las clases de la escala (Cuadro 2).

Dado que los datos de abundancia recopilados eran conteos en una unidad dada, se analizaron mediante un modelo lineal generalizado (GML) con la distribución de Poisson, para determinar la significancia de los efectos simples y la interacción. El modelo utilizado fue: 
Cuadro 2. Escala visual empleada para determinar la cobertura de las arvenses en fincas de banano y café, en los sistemas productivo convencional y orgánico. Limón y Alajuela, Costa Rica. 2015-2016.

Table 2. Visual scale used to determine weed ground cover at each surveying site in banana and coffee farms and conventional or organic production system. Limón and Alajuela, Costa Rica. 2015-2016.

\begin{tabular}{ccc}
\hline Descripción & Clases de Porcentaje de cobertura & Puntos medios de la clase \\
\hline Escasa & 0 a menos de 5 & 2,5 \\
Leve & 5 a menos de 20 & 15 \\
Moderada & 20 a menos de 50 & 37,5 \\
Alta & 50 a menos de 75 & 62,5 \\
Muy alta & 75 a 100 & 87,5 \\
\hline
\end{tabular}

Modificado de Jürgens (1985) / Moddified from Jürgens (1985).

Número de especies $=\mathrm{e}^{\mu+\beta \mathrm{i}+\gamma \mathrm{\gamma j}+\beta_{\mathrm{i}}{ }^{*} \gamma \mathrm{j}+\mathcal{E}^{\mathrm{ijk}}}$

Donde:

$\beta_{\mathrm{i}}=$ efecto del cultivo i.

$\gamma_{\mathrm{j}}=$ efecto del sistema productivo $\mathrm{j}$.

$\beta_{i}^{*} \gamma_{j}=$ efecto de interacción entre sistema productivo y cultivo.

$\varepsilon_{\mathrm{ijk}}=$ error aleatorio.

$\mathrm{i}=1,2$.

$\mathrm{j}=1,2$.

Se utilizó un nivel de significancia del 5\%. Los análisis se realizaron en JMP versión 12.

\section{Resultados}

\section{Abundancia de arvenses}

La interacción entre el cultivo y el tipo de manejo resultó significativa ( $\mathrm{p}<0,0074)$ (Cuadro 3). En banano, bajo el sistema de manejo convencional, el número de especies de arvenses fue 109\% mayor que bajo el sistema orgánico; mientras que en café esa diferencia fue del $46 \%$.

Hubo mayor abundancia de especies en el cultivo del café en comparación con el cultivo de banano. En cuanto a manejo, se encontró un menor número de plantas en la producción orgánica, tanto para el cultivo del café como para el cultivo de banano, en comparación con el sistema convencional donde se muestra que hubo mayor número de especies por sitio (Figura 1).

\section{Cobertura de arvenses}

En ambos cultivos se encontró que, en general, en el sistema de manejo orgánico se observó el mayor porcentaje de cobertura máxima, en comparación con el sistema de manejo convencional donde las arvenses se manifestaron a un nivel de cobertura menor (Cuadro 4). 
Cuadro 3. Resultados de los efectos de acuerdo con el modelo de Poisson para el cultivo de café y banano, bajo los sistemas de producción orgánica y convencional. Limón y Alajuela, Costa Rica. 2015-2016.

Table 3. Effects results according to the Poisson model for coffee and banana crops under organic and conventional production system. Limón and Alajuela, Costa Rica. 2015-2016.

\begin{tabular}{|c|c|c|c|}
\hline Efectos & Estimador & Estimación & Probabilidad \\
\hline Constante del modelo & $\mu$ & 2,5199 & 0,0001 \\
\hline Cultivo (banano) ${ }^{1}$ & $\beta$ & 0,2136 & 0,0001 \\
\hline Plaguicida (convencional) & $\gamma$ & 0,2813 & 0,0001 \\
\hline $\begin{array}{l}\text { Cultivo (banano)* }{ }^{*} \text { plaguicida } \\
\text { (convencional) }\end{array}$ & $\beta^{*} \gamma$ & 0,0933 & 0,0074 \\
\hline
\end{tabular}

${ }^{1}$ Los predictores cualitativos se codifican con 1 o - 1 por lo que la estimación para café sería -0,2136 / The qualitative predictors are codified with 1 or -1 and thus the estimation for coffee would be $-0,2136$.

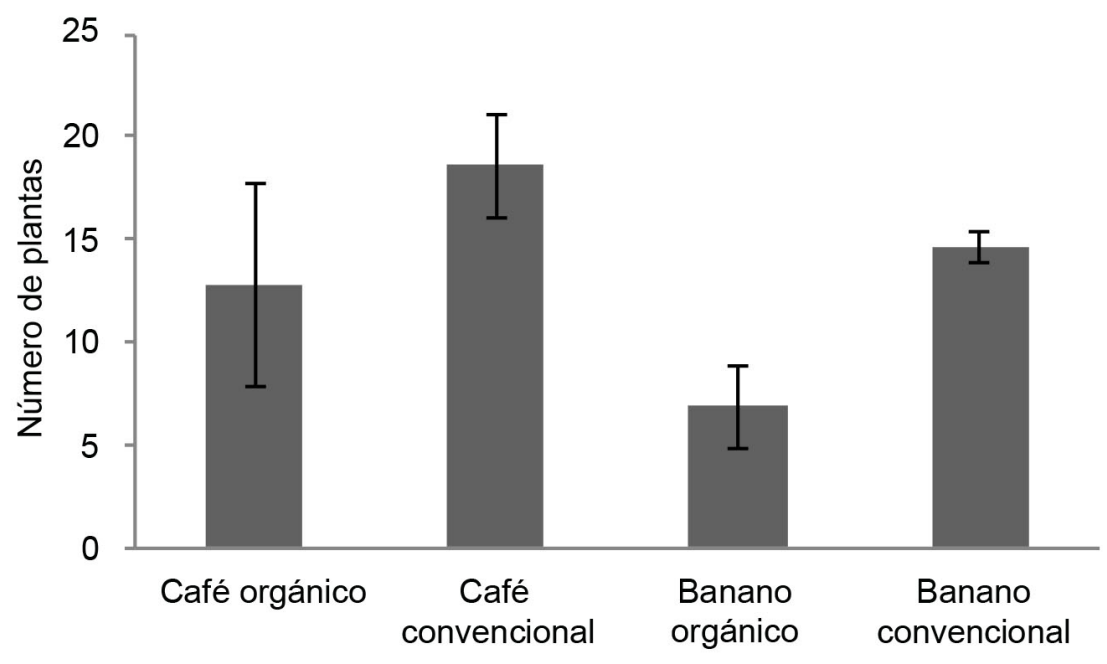

Sistema de producción por cultivo

Figura 1. Número promedio de arvenses por sitio muestreado, en los sistemas de producción orgánico y convencional, en los cultivos de café y banano. Alajuela y Limón. Costa Rica. 2015-2016.

Figure 1. Average number of weeds/site sampled, at organic and conventional production system for coffee and banana crops. Alajuela and Limón, Costa Rica. 2015-2016.

De acuerdo con los resultados encontrados, en el cultivo de café orgánico las familias de las arvenses que presentaron los valores más altos en el porcentaje promedio de cobertura máxima, fueron Commelinaceae y Convolvulaceae; en tanto que en el sistema convencional lo mostraron las familias Rubiaceae, Commelinaceae y Fabaceae. Para el caso del banano bajo el manejo orgánico, la cobertura máxima se encontró en las familias Amaranthaceae, Rubiaceae y Urticaceae, mientras que en el sistema convencional predominó la familia Amaranthaceae (Cuadro 4). 
Cuadro 4. Porcentaje de cobertura máxima promedio de cada familia de arvenses en el cultivo del café y banano bajo manejo convencional y orgánico. Limón y Alajuela, Costa Rica. 2015-2016.

Table 4. Percentage maximum ground cover average of each weed family found in coffee and banana crops under conventional and organic management. Limón and Alajuela, Costa Rica. 2015-2016.

\begin{tabular}{|c|c|c|c|c|}
\hline \multirow[t]{2}{*}{ Familia } & \multirow{2}{*}{$\begin{array}{c}\text { Café } \\
\text { Convencional }\end{array}$} & \multicolumn{3}{|c|}{ Banano } \\
\hline & & Orgánico & Convencional & Orgánico \\
\hline Amaranthaceae & 0,83 & 0,83 & 12,5 & 20,83 \\
\hline Apiaceae & 4,29 & 4,64 & 0 & 0 \\
\hline Araceae & 0 & 0 & 1,25 & 7,5 \\
\hline Aristolochiaceae & 0 & 2,5 & 0 & 0 \\
\hline Asteraceae & 1,76 & 5,74 & 1,88 & 0 \\
\hline Begoniaceae & 0 & 0 & 0 & 2,5 \\
\hline Brassicaceae & 8 & 1 & 0 & 0 \\
\hline Calceolariaceae & 1,25 & 1,25 & 0 & 0 \\
\hline Capparidaceae & 0 & 0 & 2,5 & 0 \\
\hline Caryophyllaceae & 3,75 & 3,75 & 0 & 0 \\
\hline Commelinaceae & 19,38 & 43,75 & 1,25 & 4,5 \\
\hline Costaceae & 0 & 0 & 0 & 2,5 \\
\hline Convolvulaceae & 2,5 & 62,5 & 0 & 0 \\
\hline Cucurbitaceae & 0 & 1,67 & 1,25 & 1,25 \\
\hline Cyperaceae & 6,67 & 0,83 & 1,25 & 0,63 \\
\hline Euphorbiaceae & 0 & 2,5 & 1,88 & 0,63 \\
\hline Fabaceae & 18,75 & 1,25 & 2,5 & 0 \\
\hline Loganiaceae & 0 & 0 & 2,5 & 0 \\
\hline Hypoxidaceae & 2,5 & 2,5 & 0 & 0 \\
\hline Iridaceae & 1,25 & 2,5 & 0 & 0 \\
\hline Lamiaceae & 1,25 & 1,25 & 0 & 0 \\
\hline Lytraceae & 1,25 & 1,25 & 0 & 0 \\
\hline Malvaceae & 2,5 & 0 & 0 & 0 \\
\hline Nyctinaceae & 2,5 & 0 & 0 & 0 \\
\hline Melastomataceae & 0 & 0 & 2,5 & 0 \\
\hline Oxalidaceae & 2,5 & 15 & 2,5 & 2,5 \\
\hline Passifloraceae & 2,5 & 0 & 0 & 0 \\
\hline Piperaceae & 0 & 0 & 1,67 & 1,67 \\
\hline Poaceae & 2,89 & 5,26 & 2,78 & 4,72 \\
\hline Poligonaceae & 0 & 15 & 0 & 0 \\
\hline Polygalaceae & 2,5 & 15 & 0 & 0 \\
\hline Portulacaceae & 0 & 0 & 2,5 & 0 \\
\hline Rosaceae & 2,5 & 0 & 0 & 0 \\
\hline Rubiaceae & 15,5 & 0,5 & 2,5 & 12,5 \\
\hline Scrophulariaceae & 2,5 & 2,5 & 0 & 0 \\
\hline Solanaceae & 0,63 & 1,25 & 2,5 & 0 \\
\hline Urticaceae & 0,83 & 0,83 & 1 & 20,5 \\
\hline Verbenaceae & 0 & 0 & 2,5 & 0 \\
\hline Vitaceae & 0 & 0 & 2,5 & 0 \\
\hline Total & 110,78 & 195,05 & 51,71 & 82,23 \\
\hline
\end{tabular}




\section{Discusión}

\section{Abundancia de arvenses}

En el sistema orgánico existió un menor número de especies de arvenses, dado que el control orgánico con chapias, estableció una jerarquía competitiva de unas cuantas especies dominantes, que no permitieron el establecimiento de otras menos adaptadas al sitio. En contraste, en los sistemas de producción convencional muestreados, en los cuales el control de arvenses se realizó con glifosato, se redujo de forma eficaz la biomasa de las arvenses en comparación con el uso de otros métodos de combate. No obstante, según la época de aplicación y el tipo de maleza a controlar, dicha eficacia podría reducirse (Baumgartner et al., 2007); además, el glifosato tiene poca persistencia en el suelo, debido a la inactivación que ocurre al momento de adsorberse en el mismo (Bozzo, 2010). Las causas mencionadas podrían contribuir a que otras arvenses se establezcan después de realizar la aplicación. De tal forma, en un agroecosistema en donde se efectúan aplicaciones frecuentes, con un herbicida con efecto no preemergente, el número de especies tienden a mantenerse a través del tiempo.

\section{Cobertura de arvenses}

Bajo un sistema de producción orgánica, la capacidad que tienen las diferentes especies de regenerarse es muy poca, en su lugar, existe predominancia de algunas especies, las cuales cubren el suelo. Dichas especies podrían considerarse coberturas espontáneas y pueden representar una herramienta para el manejo de arvenses en sistemas de cultivos perennes, ya que las coberturas en un sistema de producción agrícola contribuyen a la reducción de insumos agrícolas y del uso de recursos no renovables (Robačer al., 2015). El porcentaje promedio de cobertura máxima encontrado en los sistemas de manejo convencional, podría asociarse al uso del control químico, el cual cuando es empleado como único método puede contribuir a la erosión de los suelos (Hincapié-Gómez y SalazarGutiérrez, 2007).

Como posibles coberturas espontáneas en el cultivo del café se reportan las siguientes especies: Zebrina pendula (Commelinaceae), Desmodium ovalifolium, Arachis pintoi (Fabaceae) y Oxalis corniculata (Oxalidaceae) (Herrera, 1997). Bronzoni et al. (1994) menciona a Commelina diffusa (Commelinaceae), Arachis pintoi (Fabaceae), Oplismenus burmani (Poaceae) como coberturas nobles. Como coberturas espontáneas en el cultivo del banano Herrera (1997), CORBANA S.A. (2011) y Murillo et al. (2016), señalan principalmente a Geophila macropoda (Rubiaceae) y Arachis pintoi (Fabaceae). En Colombia, se reportan las siguientes arvenses como coberturas promisorias: Teramus volubilis (Fabaceae), Callisia cordifolia (Commelinaceae), Desmodium scorpiurus y D. triflorum (Fabaceae) (Carbonó y Cruz, 2005).

Las familias que componen las coberturas espontáneas reportadas en este trabajo para el cultivo del café y que coinciden con las citadas en la literatura son Commelinaceae, Rubiaceae y Fabaceae, mientras que familias como Asteraceae, Poaceae y Oxalidaceae tuvieron muy poca cobertura o nula como la que se encontró con Portulaceae. Para el cultivo del banano, la cobertura de la familia Rubiaceae fue reportada por Herrera (1997) y CORBANA (2011). La dinámica poblacional de una determinada arvense puede variar de un sitio a otro, dependiendo del manejo empleado para el control de arvenses y las condiciones climáticas prevalecientes (Abu-Dieyeh y Watson, 2007).

Aunque se ha considerado que las coberturas también puedan hacer un aporte nutricional al cultivo, como es el caso de las especies de la familia Fabaceae por su capacidad de fijar nitrógeno y luego incorporarlo al sistema productivo, según Carbonó y Cruz (2005), el aporte de nitrógeno es solo un criterio más y, en general, consideran más valioso el concepto del manejo de un complejo dinámico de especies, el cual variará con los años conforme a la dinámica poblacional de sitio y, al conocimiento que se vaya consolidando. Por otro lado, algunas especies leguminosas de los géneros Desmodium y Vigna muestran hábito trepador, lo cual dificulta su manejo (Pound, 1998). 
A diferencia del sistema convencional, donde hubo mayor número de arvenses y menor cobertura lo cual implica mayor número de especies a controlar, en el sistema orgánico se encontró menor número de arvenses pero estas a su vez presentaron una mayor cobertura en el suelo. Lo anterior representa una ventaja desde el punto de vista de manejo en un sistema perenne, por ejemplo para protección del suelo y la supresión de las especies invasoras no deseadas (Sancho y Cervantes, 1997). Por otra parte, especies adaptadas a la zona pueden ser una alternativa para uso como coberturas de origen local (Najul y Anzalone, 2006).

\section{Conclusiones}

En el presente estudio se reportaron varias familias de arvenses con potencial de cobertura en los agroecosistemas del banano y café de Costa Rica, a efectos de que esta primera aproximación sea refinada con el transcurrir de los años, conforme se acumula información sobre su uso.

En los agroecosistemas de banano y café, cuando el sistema de producción fue de tipo convencional, hubo una mayor abundancia de especies de arvenses que las encontradas en los sistemas orgánicos. Por el contrario, en el sistema de producción orgánica, la cobertura del suelo por arvenses fue mayor en ambos cultivos. Si estudios posteriores demostrasen que esa mayor cobertura por arvenses no se traduce en daños directos o indirectos a los cultivos, las ventajas se mostrarían en la protección del suelo y la eliminación del uso de herbicidas.

\section{Literatura citada}

Abu-Dieyeh, M.H., and A.K. Watson. 2007. Population dynamics of broadleaf weeds in Turfgrass as influenced by chemical and biological control methods. Weed Sci. 55:371-380. doi:10.1614/WS-06-144

Agüero, R., y S. Brenes. 2015. Software de identificación de arvenses del banano. https://play.google.com/store/apps/ details?id=edu.ucr.bananarvenses\&hl=es_419 (consultado ene.-mar. 2016).

Baumgartner, K., K.L. Steenwerth, and L. Veilleux. 2007. Effects of organic and conventional practices on weed control in a perennial cropping system. Weed Sci. 55:352-358. doi:10.1614/WS-06-171

Bozzo, M. 2010. Persistencia del glifosato y efecto de sucesivas aplicaciones en el cultivo de soja en agricultura contínua en siembra directa sobre parámetros biológicos del suelo. Tesis MSc., Universidad de la República, Montevideo, URU.

Brenes, S. 2005. Caracterización de la herbología asociada al cultivo del banano en Costa Rica. En: J.A. Sandoval, editor, Resúmenes del Primer Congreso Científico - Técnico Bananero Nacional. CORBANA. Guápiles, CRC. p. 66.

Bronzoni, G. A. Coghi, D. Cubero, J Dandois, P. Dercksen, O. Gómez, R. Ibarra, W. Mayorga, B. Sonneveld, M. Ugalde, A. Vásquez, F. Villalobos, y A Zumbado. 1994. Manual de manejo y conservación de suelos y aguas. EUNED, San José, CRC.

Carbonó, E., y Z. Cruz. 2005. Identificación de coberturas promisorias para cultivo de banano en la zona de Santa Marta, Colombia. Intropica. 2:7-22. doi:10.21676/23897864.42

CORBANA S.A. (Corporación Bananera Nacional). 2011. Implementación de buenas prácticas agrícolas para reducir el escurrimiento de plaguicidas en el cultivo del banano de la región Caribe costarricense. http://cep.unep.org/repcar/ proyectos-demostrativos/costa-rica-1/publicacionescorbana/Estudio\%20de\%20caso\%20Corbana.pdf (consultado mar. 2016). 
Cubillo, D., S. Laprade, y R. Vargas. 2001. Manual técnico para el manejo integrado de insectos plaga en el cultivo de banano. Corporación Bananera Nacional S.A., San José, CRC.

de-la-Cruz, R., E. Rojas, H. Lobón, y C. Burgos. 2001. El papel de las malezas en la reducción de la lixiviación de nutrimentos en cultivos de banano en el trópico húmedo. Manejo Integrado de Plagas. 62:29-37.

Garro, J. 2002. Plantas competidoras. Un componente más de los agroecosistemas. EUNED, San José, CRC.

Herrera, F. 1997. Plantas invasoras y su potencial de uso en agricultura tropical: coberturas en café (Coffea arabica) y banano (Musa acuminata). Bol. Tec. Est. Exp. Fabio Baudrit 30(1):51-61.

Hincapié-Gómez, E., y L.F. Salazar-Gutiérrez. 2007. Manejo integrado de arvenses en la zona cafetalera central de Colombia. Avances Técnicos 359. Centro Nacional de Investigaciones de Café (Cenicafé), Caldas, COL.

IMN (Instituto Meteorológico Nacional). 2002. Datos climáticos. https://www.imn.ac.cr/ (consultado 9 dic. 2016).

Jürgens, G. 1985. Levantamiento de malezas en cultivos agrícolas. PLITS 3(2):85-104.

Najul, C., y A. Anzalone. 2006. Control de malezas con cobertura vegetal en el cultivo de la caraota negra (Phaseolus vulgaris L.). Bioagro 18(2):75-82.

Murillo, J., V.H. Méndez-Estrada, y S. Brenes-Prendas. 2016. Efecto de Geophila macropoda (Rubiaceae) como arvense de cobertura en la erosión hídrica en bananales de Guápiles, Limón, Costa Rica. Cuadernos de Investigación UNED 8:217223.

Pinilla, C., y J. García. 2002a. Manejo integrado de malezas en plantaciones de banano. UNIBAN S.A., COL.

Pinilla, C., y J. García. 2002b. Manejo integrado de arvenses en plantaciones de banano (Musa AAA). En: Asociación de Bananeros de Colombia, editores, Memorias XV Reunión Asociación de Bananeros de Colombia. Acorbat, Medellín, COL. p. 222-235.

Pound, B. 1998. Cultivos de cobertura para la agricultura sostenible en América. FAO, Roma, ITA.

Robačer, M., S. Canali., H. Lakkenborg-Kristensen., F. Bavec., S. Grobelnik-Mlakar, M. Jakop, and M. Bavec. 2015. Cover crops in organic field vegetable production. Sci. Hort. 208:104-110. doi:10.1016/j.scienta.2015.12.029

Salazar, L.F., J.G. Arango, y C.S. Morales. 2012. Interferencia de coberturas vegetales en la zona de raíces y entre calles del cultivo del café. Cenicafé 63(2):50-57.

Sancho, F., y C. Cervantes. 1997. El uso de plantas de cobertura en sistemas de producción de cultivos perennes y anuales en Costa Rica. Agron. Costarricense 21(1):111-120.

Wielemaker, F.1997. Banana production with "Pinto`s peanut" (Arachis pintoi cv amarillo) and "Oreja de ratón" (Geophila repens) as cover crops. En: J. Yglesias, editor, Memoria primer taller international de control biológico y producción integrada en el cultivo de banano. EARTH, San José, CRC. p. 84-89. 